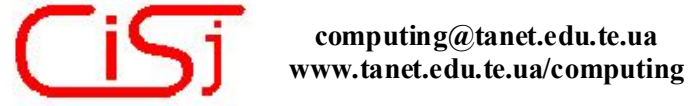

\title{
AUTOMATIC MICROINJECTION SYSTEM USING STEREOSCOPIC MICROSCOPE
}

\author{
Junko Sakiyama ${ }^{1)}$, Hideki Yamamoto ${ }^{2)}$

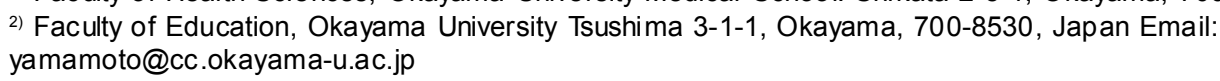

2) Faculty of Education, Okayama University Tsushima 3-1-1, Okayama, 700-8530, Japan Email:

yamamoto@cc.okayama-u.ac.jp \\ 1) Faculty of Health Sciences, Okayama University Medical School. Shikata 2-5-1, Okayama, 700-8558, Japan
}

\begin{abstract}
In this paper, we describe a visual feedback system using a stereoscopic microscope that controls a micromanipulator so that a needle head may pierce a target as much length as desired. At first, we developed an image processing algorithm for the tip of needle head to touch the target. Secondarily, we developed an algorithm for prediction of the tip position of the needle head within the target. By performing a preoperation, the shape of the needle head is preserved as a reference pattern. When the needle head piercing the target, the shape of the needle head within the target is predicted by pattern matching. Thus, we developed a microinjection system that axially pierces the target. Experimental results show that the proposed system may be useful in micromanipulation such as microinjection to brain areas in neuroanatomy.
\end{abstract}

\section{Keywords: - Microinjection, Micromanipulation, Stereoscopic microscope, Image processing}

\section{INTRODUCTION}

In the area of biotechnology, a microinjection system is widely used for such purposes as operating on genes and transferring biological materials into cells. For some experiments, such as biochemical assay, a large number of cells have to be injected in a short time. The development of a computer-assisted and microprocessor-controlled injection system provides high injection rates. The AIS(automated injection system, Zeiss, Germany) is an automated microinjection system, and it permits reliable and simple microinjection into living cells by a system configuration of various hardware and software components that controls the injection procedure [1]. However, the AIS cannot perform sufficiently with the cells in various thickness because the manipulator of the AIS moves constantly. For achieving the flexible microinjection, it is necessary to develop an automatic microinjection system that allows control of the injector so that it may pierce the cells in the desired depth.

Recently, the several techniques of visual feedback are proposed[2,3]. However, these systems have some problems in performing the microinjection. At first, it has difficulties to measure the distance between the injector and the cell under the stereoscopic microscope. Secondarily, the significant problem is that the tip position of the needle head disappears when the needle head pierces the cell. By these reasons, it is difficult to estimate that the injector pierces the cell in the desired depth.

In this paper, we propose an automatic microinjection system under the stereoscopic microscope. By using the stereovision method, we can measure the distance between the tip of the needle head attached to the micromanipulator and the target on the stage of stereoscopic microscope. We develop prediction algorithms of the needle head to pierce the target as much length as desired. Experimental results show that the proposed system may be useful in microinjection.

\section{METHODS}

\subsection{Three dimensional image meas urement}

Figure 1 shows the microinjection system. The system consists of a stereoscopic microscope, two CCD cameras, a micromanipulator, and a personal computer. A target is set on the stage of the microscope. The cameras are mounted to the eyepieces of the microscope. The micromanipulator has three degrees of freedom. It moves linearly. The needle is attached to the micromanipulator. The left image and the right image are inputted to the personal computer that performs image processing to detect the needle head and the target. We use rectangular $(\mathrm{X}, \mathrm{Y}, \mathrm{Z})$ coordinates. The $\mathrm{X}$ axis and $\mathrm{Z}$ axis are parallel to the stage. The $Y$ axis is vertical to the stage of the microscope. The distance between the tip position of the needle head and the 


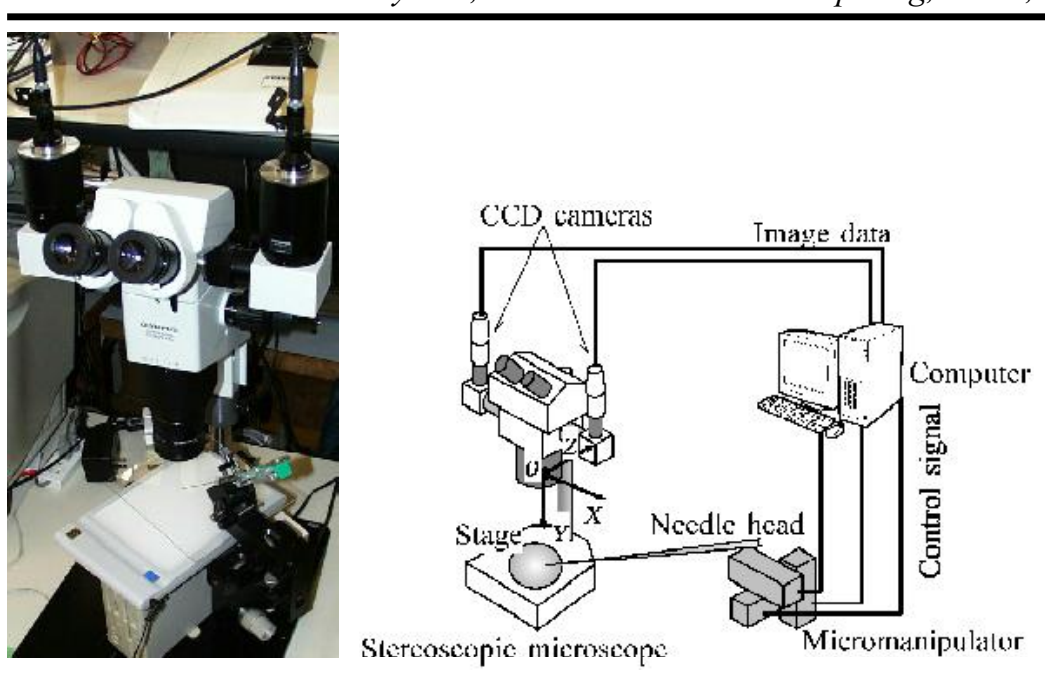

Fig. 1 - Microinjection system.

of the needle head becomes round as shown in the figure $3 \mathrm{~b}$ ). We make it clear that the tip size $\mathrm{S}$ which is before piercing becomes larger than the tip size $\mathrm{S}$ which is after piercing.

\subsection{Detection of the tip position of the needle head}

After the tip of the needle head is in the target, the needle head proceeds to the goal. When the needle head pierces the target, the tip of the needle head becomes invisible as shown in figure 4 . When the needle head pierces the target to the depth de-

target is measured three dimensionally by the stereovision method $[4,5,6]$. We have made it clear that the distance between the needle head and the object lens of the stereoscopic microscope is given by a linear equation.

$$
y=A\left(x_{l}-x_{r}\right)+B
$$

where $x_{l}$ represents the point of the needle head in the left camera and $x_{r}$ represents the points of the needle head in the right camera, respectively. The distance $\left(x_{l}-x_{r}\right)$ is called a position difference. The needle head is guided to reach the target.

\subsection{Path design of the needle head}

We develop a moving strategy of the needle head to pierce the target as much length as desired[7,8]. Figure 2 shows the path of the needle head. We decide the position of a goal having an acceptable limit such as a defined goal zone D. At first, the needle head approaches to the target. The distance between the needle head and the target is measured three dimensionally. Secondarily, the needle head descends vertically until the needle head pierces the target. As the target is elastic, the surface of the target is distorted by the needle head when the needle head touch the target. To avoid that the needle head makes the target slip, the needle head descends vertically until the tip of the needle head pierces the target. Therefore, it is necessary to detect the instance that the needle head begins to pierce the target. Figure 3 shows length I and size $\mathrm{S}$ of the tip area of the needle head. Before the needle head pierces the target, the shape of the needle head is sharp as shown in figure $3 \mathrm{a}$ ). After the needle head piercing the target, a part of the tip of the needle head is hidden and the shape sired, the remaining part of the needle head, which is called a visible area is detected by the pattern matching $[3,4]$. Figure 5 shows that the needle head is obliquely attached to the micromanipulator. In this figure, the length $\mathrm{R}$ is given by

$$
R=P \cos \theta
$$

where, $P$ is an axial piercing length and $R$ is the same length in the $\mathrm{XZ}$ plane as shown in figure 4 .

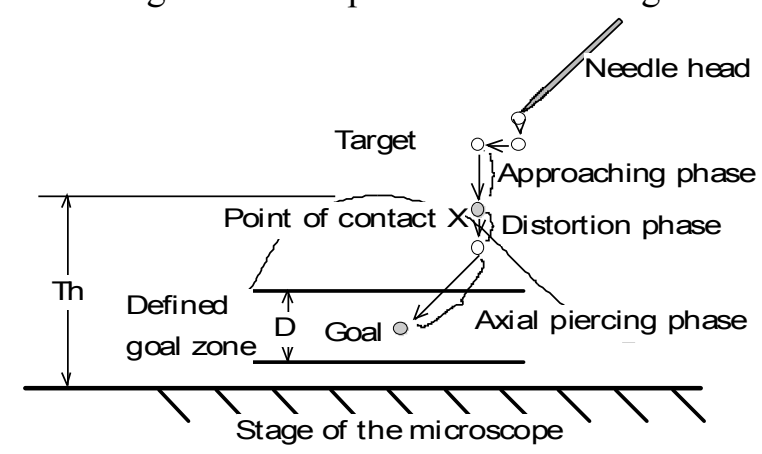

Fig. 2 - Path of the needle head.
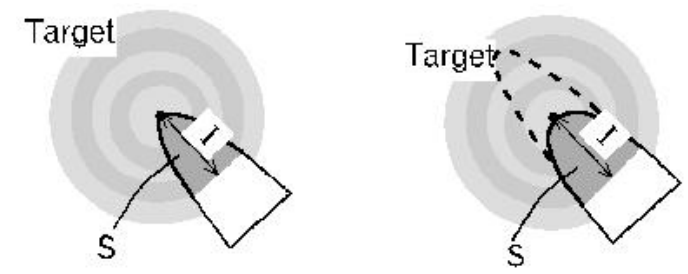

a) Needle head before piercing

b) Needle head after piercing

Fig. 3 - Length I and Size S of the tip area.

\subsection{Preoperation to get the reference pattern}

As mentioned above, it is necessary to get the reference pattern in order to pierce the target at a high accuracy. We perform a preoperation before the piercing the target. Figure 6 shows a preoperation to get the reference pattern. In this 
figure, the needle head is moved to the same height of the goal. After the needle head is detected, a square region of interest is automatically set as shown in figure 4 . In this figure, the tip position of the needle head is on a diagonal line of the square region. According to the thickness of the target, we usually decide the piercing length $P$ in figure 5. Therefore, the length $R$ in figure 4 is given by the equation (2). And the visible area of the needle head is used as the reference pattern.

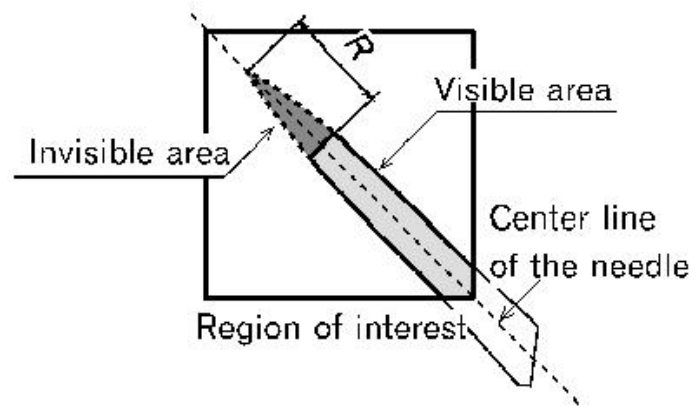

Fig. 4 - Reference pattern.

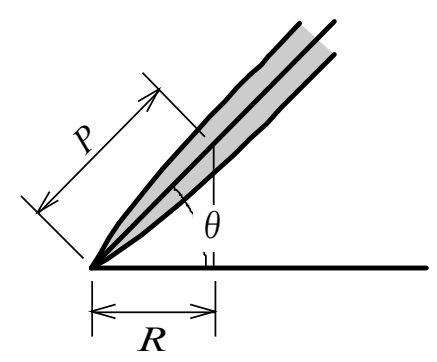

Fig. 5 - Length of the needle head piercing the target.

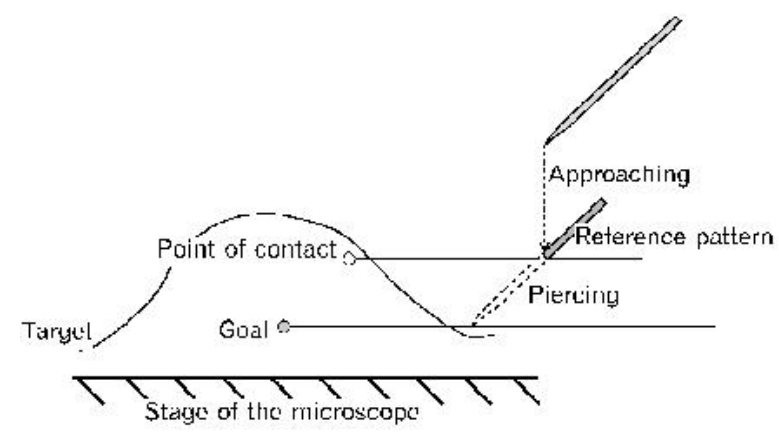

Fig. 6 - Preoperation for piercing the target.

\subsection{Axial piercing as much length as desired}

When the needle head pierces the target, the visible part of the needle head corresponding to the reference pattern is searched. The centerline of the needle head is easily obtained by image processing. We set regions of interest on the center line of the needle head for the pattern matching. Upper left corner and lower right corner of the re- gion of interest are on the center line. In order to detect the position of the reference pattern on the needle head, we calculated the correlations $R_{k}$ between the reference pattern and the regions of interest $[3,4]$. The position of the reference pattern is detected on the center line LL' as shown in figure 7 , where the correlation is closest to 1.0. If there is a length $\mathrm{H}$ as shown in figure 7, the length $\mathrm{H}$ indicates the remaining length to pierce the target. In this way, the needle head is gradually moved so that distance $\mathrm{H}$ may become to be zero.

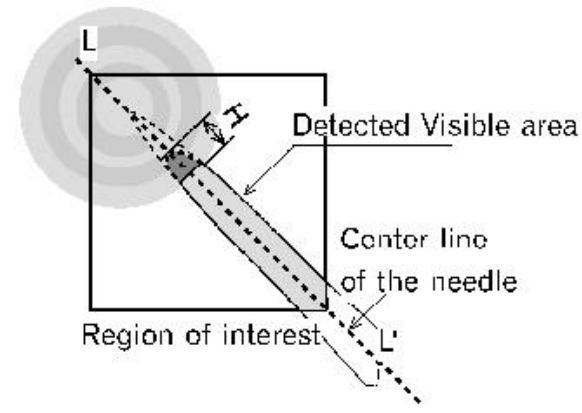

Fig. 7 - Search method of the tip position of the
needle head within the target.

\section{RESULTS}

The magnification of the stereoscopic microscope is 10 times. A steel needle is attached to a micromanipulator. At first, the relation between the distance from the object lens of the microscope to the tip position of the needle head and the position difference of the left and right images is obtained as shown in figure 8 . We make clear that the distance of the needle head can be approximated by the equation (1). The value of $\mathrm{A}$ and $\mathrm{B}$ of equation (1) are -0.0366 and 83.6271 , respectively. Measurement resolution in $\mathrm{Y}$ axis is given by the value of the coefficient A. Table 1 shows measurement resolutions at 10 times magnification rate.

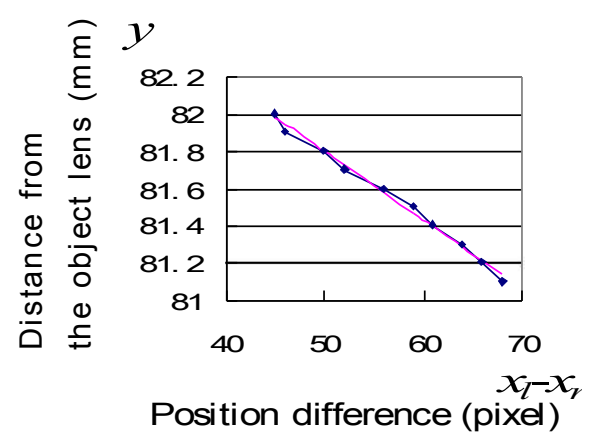

Fig. 8 - Distance and position difference.

A leaf is chosen as a target. An experiment is performed so that the needle head pierces the target and reach to a goal, which is appointed on the 
center of a vein of the leaf as shown in figure 9 . The vein of the leaf is $1.5 \mathrm{~mm}$ in diameter, and the piercing length $P$ is set as $0.5 \mathrm{~mm}$. The needle head has an angle toward the stage of the microscope. By using the equation (2), the value of $R$ becomes to be $0.35 \mathrm{~mm}$. Thus, we can decide the position of point of contact $\mathrm{X}$ as mentioned in figure 2. Here, the defined goal zone $\mathrm{D}$ is set as $0.5 \mathrm{~mm}$.

\section{Table 1. Measurement resolutions} (10 times of magnification)

$\begin{array}{ll}\mathrm{X} \text { and } \mathrm{Z} \text { axes } & 0.0096 \mathrm{~mm} / \mathrm{pixel} \\ \mathrm{Y} \text { axis } & 0.0366 \mathrm{~mm} / \mathrm{pixel} \\ \end{array}$

As the leaf, as a target, has elasticity, the surface of the leaf becomes to distort when the needle head contacts and begins to pierce. Figure 10 shows the tip size of the needle head. Here, the value of I is 30 pixels. The tip size of the needle head before piercing is about 604 pixels. Standard deviation is about 19 pixels. If the size of tip area becomes more than about 623 pixels, the needle head is considered to pierce the target.

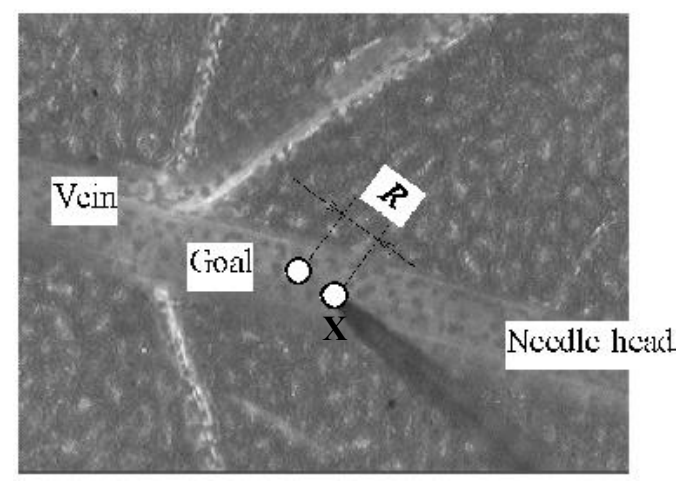

Fig . 9 - Goal.

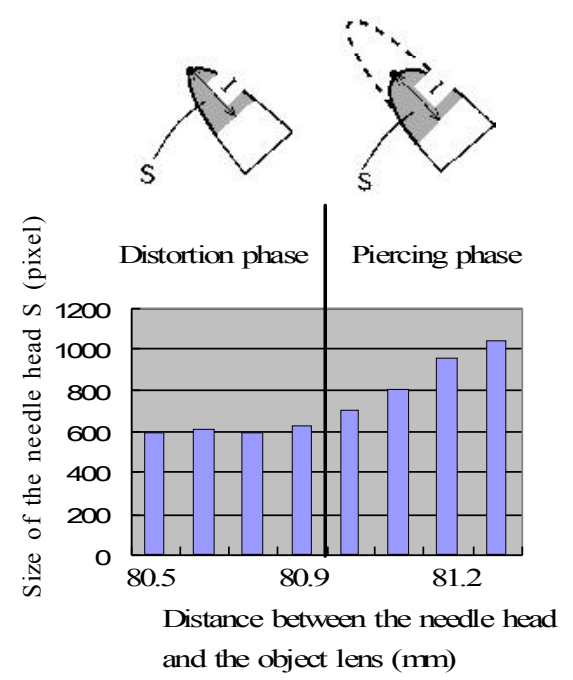

Fig. 10 - Size of the tip of the needle head
Figure 11 shows the needle head in the preoperation experiment. A region of interest is set to the tip of the needle head. And the reference pattern is obtained as shown in figure $11 \mathrm{~b}$ ).

a) Needle head approaching the target

b) Reference pattern

Fig. 11 - Needle head piercing the target.

Figure 12 shows the needle head when piercing the target at desired length. The tip area of the needle head is hidden and point $\mathrm{P}^{\prime}$ is the tip position of the reference pattern. Figure 13 shows the correlation values on the center line of the needle head, which becomes maximum at the point P'. Figure 14 shows that the needle head pierces the target $0.44 \mathrm{~mm}$ in length. In this way, the tip position of the needle head within the target can be fully controlled by the micromanipulator.

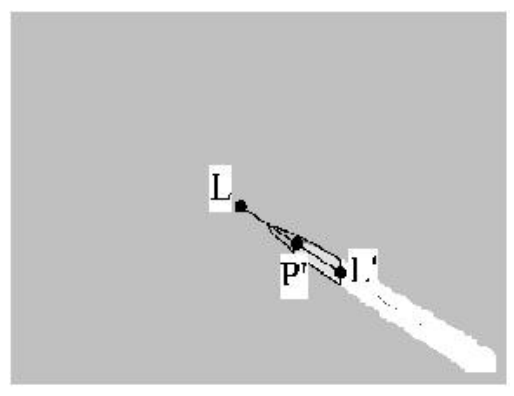

Fig. 12 - Predicted needle head.

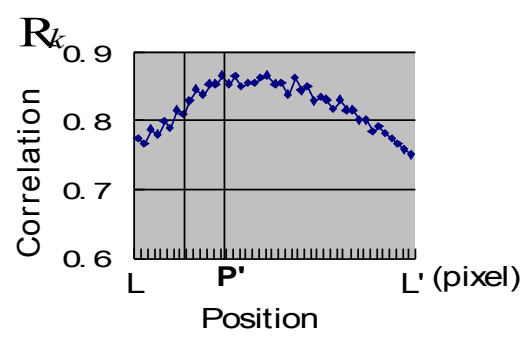

Fig. 13 - The values of correlation.

\section{CONCLUSIONS}

In this paper, an automatic microinjection system with a stereoscopic microscope is proposed. A method for prediction of the tip position of the 
needle head within the target is developed. During the needle head proceeds within the target, the tip position of the needle head is predicted by the pattern matching. As a result, the tip position of the needle head within the target is fully controlled and effectively guided to reach a goal within the target. Experimental results show that the proposed system may be useful in microinjection of brain areas in the neuroanatomy.

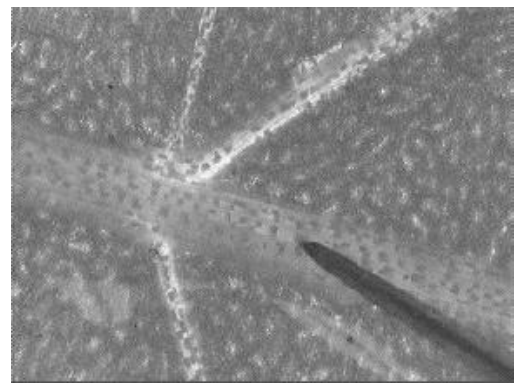

Fig. 14 - Needle head piercing the target $0.44 \mathrm{~mm}$ in length.

\section{REFERENCES:}

[1] W. Ansorge, R. Saferich. : Microinjection and Transgenesis Strategies and Protocols. Springer Lab Manual, 31-46, 1998.

[2] J.T. Feddeman, G.C.S. Lee, O.R. Mitchell. Model-based Visual Feedback Control for HandEye Coordinated Robotic System. IEEE Computer, 21-31, 1992.

[3] A. Castano, S. Hutchinson. Visual compliance: Task-directed visual servo control. IEEE Transactions on Robotics and Automation, 10(3):334-341, 1994.

[4] T. Sano H. Nagahata, H. Yamamoto : Automatic Micromanipulation System Using Stereoscopic Microscope. Proceedings of the $16^{\text {th }}$ IEEE Instrumentation and Measurement Technology Conference, IMTC '99, 1:327-331, 1999.

[5] T. Sano, H. Yamamoto : Micromanipulation using Stereoscopic Microscope. Proceedings of the IEEE International Symposium on Industrial Electronics, ISIE'99, 3:1066-1069, 1999.

[6] T. Sano, H. Yamamoto : Study of Micromanipulation using Stereoscopic Microscope, Proceedings of the $17^{\text {th }}$ IEEE Instrumentation and Measurement Technology Conference, IMTC/2000, 3:1227-1231, 2000.

[7] J. Sakiyama, M. Okamoto, H. Yamamoto : Visual Feedback System for Neuroanatomy, Proceedings of $16^{\text {th }}$ World Congress of International Measurement Confederation, 1-4, 2000.

[8] J. Sakiyama, M. Okamoto, H. Yamamoto : A Fluorescent Tracing of Hilus-Granular Organization Utilizing Visual Feedback System, Proceed- ings of the $18^{\text {th }}$ IEEE Instrumentation and Measurement Technology Conference, IMTC/2001, 1:356-360, 2001.

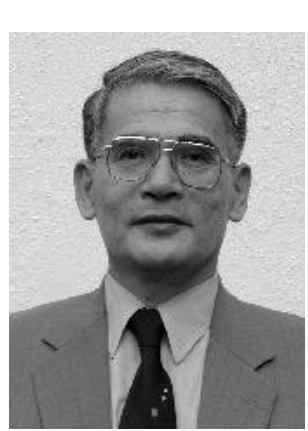

Hideki Yamamoto was born in 1944. He received the B.S. degree in electrical engineering from Okayama University, Okayama, Japan, in 1966, and the Ph.D. degree in electrical engineering from Kyoto University, Kyoto, Japan, in 1983.

$\mathrm{He}$ is currently a Professor in the Department of Education at Okayama University. His research interests include image processing and signal processing. 